Canad. Math. Bull. Vol. 62 (2), 2019 pp. 349-354

http://dx.doi.org/10.4153/CMB-2018-023-7

(C) Canadian Mathematical Society 2018

\title{
Jordan-Chevalley Decomposition in Lie Algebras
}

\author{
Leandro Cagliero and Fernando Szechtman
}

Abstract. We prove that if $\mathfrak{s}$ is a solvable Lie algebra of matrices over a field of characteristic 0 and $A \in \mathfrak{s}$, then the semisimple and nilpotent summands of the Jordan-Chevalley decomposition of $A$ belong to $\mathfrak{s}$ if and only if there exist $S, N \in \mathfrak{s}, S$ is semisimple, $N$ is nilpotent (not necessarily $[S, N]=0)$ such that $A=S+N$.

\section{Introduction}

All Lie algebras and representations considered in this paper are finite dimensional over a field $\mathbb{F}$ of characteristic 0 . An important question concerning a given representation $\pi: \mathfrak{g} \rightarrow \mathfrak{g l}(V)$ of a Lie algebra $\mathfrak{g}$ is ( $c f$. [B2, Ch. VII, $\$ 5])$

$(*)$ Does $\pi(\mathfrak{g})$ contain the semisimple and nilpotent parts of the Jordan-Chevalley decomposition (JCD) in $\mathfrak{g l}(V)$ of $\pi(x)$ for a given $x \in \mathfrak{g}$ ?

For semisimple Lie algebras, this is true for any representation and this classic result is a cornerstone of the representation theory of semisimple Lie algebras (see [Hu, $\$ 6.4$ and $\mathrm{Ch}$. VI] or [FH, $\$ 9.3$ and Ch. 14]). We are interested in the classification of indecomposable representations of certain families of non semisimple Lie algebras (see [CS2, CS3]), and an extension of the classical result to more general Lie algebras will prove useful in this endeavour. In a different direction, the recent article [Ki], studies the existence of a Jordan-Chevalley-Seligman decomposition in prime characteristic.

The question $(*)$ led us to study the existence and uniqueness of abstract JCD's in arbitrary Lie algebras [CS]. Recall that an element $x$ of a Lie algebra $\mathfrak{g}$ is said to have an abstract JCD if there exist unique $s, n \in \mathfrak{g}$ such that $x=s+n,[s, n]=0$ and given any finite dimensional representation $\pi: \mathfrak{g} \rightarrow \mathfrak{g l}(V)$ the JCD of $\pi(x)$ in $\mathfrak{g l}(V)$ is $\pi(x)=\pi(s)+\pi(n)$. The Lie algebra $\mathfrak{g}$ itself is said to have an abstract JCD if every one of its elements does. The main results of [CS] are Theorems 1 and 2, and they respectively state that $a$ Lie algebra has an abstract JCD if and only if it is perfect, and an element of a Lie algebra $\mathfrak{g}$ has an abstract JCD if and only if it belongs to $[\mathfrak{g}, \mathfrak{g}]$. These theorems, though related to question $(*)$, do not provide a satisfactory answer to it.

The purpose of this note is two-fold: on one hand we prove Theorem 1.1 below, which directly addresses question $(*)$ and allows us to derive from it [CS, Theorems 1 and 2]. On the other hand, we recently realized that there is a gap in the original

Received by the editors January 8, 2018; revised May 14, 2018.

Published electronically July 23, 2018.

Author L. C. was supported in part by CONICET and SECYT-UNC grants.

Author F. S. was supported in part by an NSERC discovery grant.

AMS subject classification: 17-08, 17B05, 20C40, 15A21.

Keywords: solvable Lie algebra, Jordan-Chevalley decomposition, representation. 
proof of [CS, Theorems 1 and 2], since [CS, Lemma 2.1] is not true. Therefore, we leave [CS, Theorems 1 and 2] in good standing by giving a correct proof derived from Theorem 1.1.

Theorem 1.1 Let $\mathfrak{s}$ be a solvable Lie algebra of matrices, let $A \in \mathfrak{s}$, and assume that $A=S+N$ with $S, N \in \mathfrak{s}, S$ semisimple, $N$ nilpotent (we are not assuming $[S, N]=0$ ). Then the semisimple and nilpotent summands of the JCD of A belong to $\mathfrak{s}$.

This theorem is a consequence of the following result.

Theorem 1.2 Let $\mathbb{F}$ be algebraically closed. Given a square matrix $A=S+N$ with $S$ semisimple and $N$ nilpotent, let $\left\{S_{n}\right\}$ and $\left\{N_{n}\right\}$ be sequences defined inductively by

$$
S_{0}=S \text { and } N_{0}=N \text {, }
$$

and, if $\left[S_{n}, N_{n}\right] \neq 0$, let $\left(N_{n}\right)_{\lambda_{n}}$ be a non-zero eigenmatrix of $\operatorname{ad}\left(S_{n}\right)$ corresponding to a non-zero eigenvalue $\lambda_{n}$ appearing in the $\operatorname{ad}\left(S_{n}\right)$-decomposition of $N_{n}$, and let

$$
S_{n+1}=S_{n}+\left(N_{n}\right)_{\lambda_{n}} \text { and } N_{n+1}=N_{n}-\left(N_{n}\right)_{\lambda_{n}} .
$$

(The sequences depend on the choice of the non-zero eigenvalues.)

If $\{S, N\}$ generates a solvable Lie algebra $\mathfrak{s}$, then (independently of the choice of the eigenvalues)

(i) $S_{n}$ is semisimple, $N_{n}$ is nilpotent, and $S_{n}, N_{n} \in \mathfrak{s}$ for all $n$,

(ii) there is $n_{0}$ such that $\left[S_{n_{0}}, N_{n_{0}}\right]=0$.

In particular, $A=S_{n_{0}}+N_{n_{0}}$ is the Jordan-Chevalley decomposition of $A$ with both components $S_{n_{0}}, N_{n_{0}} \in \mathfrak{s}$. Moreover, if $\pi: \mathfrak{s} \rightarrow \mathfrak{g l}(V)$ is a representation such that $\pi(S)$ is semisimple and $\pi(N)$ is nilpotent, then $\pi(A)=\pi\left(S_{n_{0}}\right)+\pi\left(N_{n_{0}}\right)$ is the JordanChevalley decomposition of $\pi(A)$.

\section{Jordan-Chevalley Decomposition of Upper Triangular Matrices}

This section is devoted to proving Theorem 1.2, and thus we assume that $\mathbb{F}$ is algebraically closed. Let $\mathfrak{t}$ denote the Lie algebra of upper triangular $n \times n$ matrices over $\mathbb{F}$, let $\mathfrak{t}^{\prime}=[\mathfrak{t}, \mathfrak{t}]$, and let $\mathfrak{s}$ be a Lie subalgebra of $\mathfrak{t}$.

Lemma 2.1 Let $S, X, N \in \mathfrak{s}$ and assume that $\operatorname{ad}_{\mathfrak{s}}(S)(N)=\lambda N$, with $\lambda \in \mathbb{F}$, and $\operatorname{ad}_{\mathfrak{s}}(S)(X)=\mu X$, with $0 \neq \mu \in \mathbb{F}$ (in particular, $X \in \mathfrak{t}^{\prime}$ ). Then

$$
\exp \left(-\mu^{-1} \operatorname{ad}_{\mathfrak{s}}(X)\right)(N)=\sum_{j=0}^{n-1} \frac{(-\mu)^{-j}}{j !} \operatorname{ad}_{\mathfrak{s}}(X)^{j}(N)
$$

is an eigenmatrix of $\operatorname{ad}_{\mathfrak{s}}(S+X)$ of eigenvalue $\lambda$, and it belongs to $\mathfrak{s}$. In particular, $S$ is semisimple if and only if $S+X$ is semisimple. 
Proof Since $X \in \mathfrak{t}^{\prime}$, we see that $-\mu^{-1} \mathrm{ad}_{\mathfrak{s}}(X)$ is a nilpotent derivation of $\mathfrak{s}$, so $\exp \left(-\mu^{-1} \operatorname{ad}_{\mathfrak{s}}(X)\right) \in \operatorname{Aut}(\mathfrak{s})$. In particular, $\exp \left(-\mu^{-1} \operatorname{ad}_{\mathfrak{s}}(X)\right)(N) \in \mathfrak{s}$ and

$$
\begin{aligned}
& {[\exp }\left.\left(-\mu^{-1} \operatorname{ad}_{\mathfrak{s}}(X)\right)(S), \exp \left(-\mu^{-1} \operatorname{ad}_{\mathfrak{s}}(X)\right)(N)\right] \\
& \quad=\exp \left(-\mu^{-1} \operatorname{ad}_{\mathfrak{s}}(X)\right)([S, N]) \\
& \quad=\lambda \exp \left(-\mu^{-1} \operatorname{ad}_{\mathfrak{s}}(X)\right)(N) .
\end{aligned}
$$

But $[S, X]=\mu X$ yields $\exp \left(-\mu^{-1} \operatorname{ad}_{\mathfrak{s}}(X)\right)(S)=S+X$, so $\exp \left(-\mu^{-1} \operatorname{ad}_{\mathfrak{s}}(X)\right)(N)$ is an eigenmatrix of $\operatorname{ad}_{\mathfrak{s}}(S+X)$ of eigenvalue $\lambda$. Consequently, if $\operatorname{ad}_{\mathfrak{t}}(S)$ is semisimple then $\exp \left(-\mu^{-1} \operatorname{ad}_{\mathfrak{t}}(X)\right)$ transforms a basis of eigenmatrices of $\operatorname{ad}_{\mathfrak{t}}(S)$ into a basis of eigenmatrices of $\operatorname{ad}_{\mathfrak{t}}(S+X)$.

To complete the proof it is sufficient to show that a matrix $A \in \mathfrak{t}$ is semisimple if and only if $\operatorname{ad}_{\mathfrak{t}}(A)$ is semisimple. The 'only if' part is clear. Conversely, if $\operatorname{ad}_{\mathfrak{t}}(A)$ is semisimple and $A=A_{s}+A_{n}$ is the JCD of $A$, then $A_{s}, A_{n} \in \mathfrak{t}$ (both are polynomials in $A$ ), and it follows that $\operatorname{ad}_{\mathfrak{t}}(A)=\operatorname{ad}_{\mathfrak{t}}\left(A_{s}\right)+\operatorname{ad}_{\mathfrak{t}}\left(A_{n}\right)$ is the JCD of ad $\mathrm{d}_{\mathfrak{t}}(A)$. By uniqueness, $\operatorname{ad}_{\mathfrak{t}}\left(A_{n}\right)=0$, and this implies that $A_{n}=0$, since $A_{n} \in \mathfrak{t}^{\prime}$ and the centralizer of $\mathfrak{t}$ in $\mathfrak{t}^{\prime}$ is 0 .

Let $S \in \mathfrak{s}$ be semisimple. Let $\Lambda$ be the set of eigenvalues of $\operatorname{ad}_{\mathfrak{s}}(S)$, and for each $\lambda \in \Lambda$, let $\mathfrak{s}_{\lambda} \subset \mathfrak{s}$ be the corresponding eigenspace. Given $N \in \mathfrak{s}$, let

$$
N=\sum_{\lambda \in \Lambda} N_{\lambda},
$$

where each $N_{\lambda} \in \mathfrak{s}_{\lambda}$. We refer to the above as the $\operatorname{ad}_{\mathfrak{s}}(S)$-decomposition of $N$.

For $k=0, \ldots, n-1$, let $\mathfrak{t}_{k}$ be the subspace of $\mathfrak{t}$ consisting of those matrices whose non-zero entries lay only on the diagonal $(i, j)$ such that $j-i=k$. Given $N \in \mathfrak{t}$, let $d_{k}(N) \in \mathfrak{t}_{k}$ be defined so that $N=\sum_{k=0}^{n-1} d_{k}(N)$. We now introduce a function that will used to measure how close two matrices are to commuting with each other.

Definition 2.2 Let $S, N \in \mathfrak{s}$, with $S$ semisimple, and let $N=\sum_{\lambda \in \Lambda} N_{\lambda}$ be the decomposition of $N$ as a sum of eigenmatrices of $\operatorname{ad}_{\mathfrak{s}}(S)$. For $k=0, \ldots, n-1$, let

$$
C_{S, k}(N)=\left\{\lambda \in \Lambda: \lambda \neq 0 \text { and } d_{k}\left(N_{\lambda}\right) \neq 0\right\},
$$

let $c_{S, k}(N)$ be the number of elements in $C_{S, k}(N)\left(c_{S, 0}(N)=0\right.$, since $\lambda \neq 0$ implies that $\left.N_{\lambda} \in \mathfrak{t}^{\prime}\right)$, and let

$$
\gamma_{S}(N)=\left(c_{S, 1}(N), \ldots, c_{S, n-1}(N)\right) \in \mathbb{Z}_{\geq 0}^{n-1} .
$$

It is clear that $c_{S, k}(N) \leq \operatorname{dim} \mathfrak{s}$ for all $k$ and $[S, N]=0$ if and only if $\gamma_{S}(N)=$ $(0, \ldots, 0)$.

Lemma 2.3 Let $S, X, N \in \mathfrak{s}$ with $S$ semisimple and $\operatorname{ad}_{\mathfrak{s}}(S)(X)=\mu X$, with $0 \neq \mu \in \mathbb{F}$. Let $k_{0} \geq 1$ be the lowest $k$ such that $d_{k}(X) \neq 0\left(\mu \neq 0\right.$ implies $X \in \mathfrak{t}^{\prime}$ and hence $\left.k_{0} \geq 1\right)$. Then $C_{S+X, k}(N)=C_{S, k}(N)$ for all $k \leq k_{0}$.

Proof We first point out that it follows from Lemma 2.1 that $S+X$ is semisimple, and thus it makes sense to consider $C_{S+X, k}(N)$. 
Let

$$
N=\sum_{\lambda \in \Lambda} N_{\lambda}, \quad N_{\lambda} \in \mathfrak{s},
$$

be the $\operatorname{ad}_{\mathfrak{s}}(S)$-decomposition of $N$. Let

$$
\widetilde{N}_{\lambda, 0}=\exp \left(-\mu^{-1} \operatorname{ad}_{\mathfrak{s}}(X)\right)\left(N_{\lambda}\right),
$$

and, for $j \geq 1$, let $\widetilde{N}_{\lambda, j}=\frac{\mu^{-j}}{j !} \operatorname{ad}_{\mathfrak{s}}(X)^{j}\left(\widetilde{N}_{\lambda, 0}\right)$.

It follows from Lemma 2.1 that $\widetilde{N}_{\lambda, j}$ is an eigenmatrix of $\operatorname{ad}_{\mathfrak{s}}(S+X)$ of eigenvalue $\lambda+j \mu$. Since

$$
N_{\lambda}=\exp \left(\mu^{-1} \operatorname{ad}_{\mathfrak{s}}(X)\right)\left(\widetilde{N}_{\lambda, 0}\right)=\widetilde{N}_{\lambda, 0}+\widetilde{N}_{\lambda, 1}+\widetilde{N}_{\lambda, 2}+\cdots,
$$

it follows that

$$
N=\sum_{\lambda \in \Lambda} \sum_{j \geq 0} \widetilde{N}_{\lambda, j}=\sum_{\lambda \in \Lambda} \widetilde{N}_{\lambda, 0}+\sum_{\lambda \in \Lambda} \sum_{j \geq 1} \widetilde{N}_{\lambda, j}
$$

and this leads to the decomposition of $N$ as a sum of eigenmatrices of $\operatorname{ad}_{\mathfrak{s}}(S+X)$ (after adding up those $\widetilde{N}_{\lambda, j}$ with the same eigenvalue).

Let $k \leq k_{0}$ (recall that $k_{0}$ is the lowest $k$ such that $d_{k}(X) \neq 0$ ). Since $k_{0} \geq 1$, it follows that

$$
d_{k}\left(\widetilde{N}_{\lambda, j}\right)= \begin{cases}d_{k}\left(N_{\lambda}\right) & \text { if } j=0 \\ 0 & \text { if } j \geq 1\end{cases}
$$

This implies $C_{S+X, k}(N)=C_{S, k}(N)$.

Lemma 2.4 Let $S, N \in \mathfrak{s}$, with $S$ semisimple, and let $N=\sum_{\lambda \in \Lambda} N_{\lambda}$ be the $\operatorname{ad}_{\mathfrak{s}}(S)$-decomposition of $N$. Assume that there is $\lambda_{0} \in \Lambda$ with $\lambda_{0} \neq 0$ such that $N_{\lambda_{0}} \in \mathfrak{s}_{\lambda_{0}}$ is non-zero. Then

$$
\gamma_{S+N_{\lambda_{0}}}\left(N-N_{\lambda_{0}}\right)<\gamma_{S}(N)
$$

in the lexicographical order. (The pair $\left(S+N_{\lambda_{0}}, N-N_{\lambda_{0}}\right)$ is closer to commuting than the pair $(S, N)$.)

Proof Let $k_{0}$ be the lowest $k$ such that $d_{k}\left(N_{\lambda_{0}}\right) \neq 0\left(k_{0} \geq 1\right.$, since $\left.N_{\lambda_{0}} \in \mathfrak{t}^{\prime}\right)$. It is clear that

$$
c_{S, k}\left(N-N_{\lambda_{0}}\right)= \begin{cases}c_{S, k}(N) & \text { if } k<k_{0}, \\ c_{S, k_{0}}(N)-1 & \text { if } k=k_{0},\end{cases}
$$

and thus $\gamma_{S}\left(N-N_{\lambda_{0}}\right)<\gamma_{S}(N)$.

It follows from Lemma 2.3 that, for $k \leq k_{0}$,

$$
c_{S+N_{\lambda_{0}}, k}\left(N-N_{\lambda_{0}}\right)=c_{S, k}\left(N-N_{\lambda_{0}}\right),
$$

and this, combined with (2.1), implies $\gamma_{S+N_{\lambda_{0}}}\left(N-N_{\lambda_{0}}\right)<\gamma_{S}(N)$ in the lexicographical order.

We are now in a position to prove Theorem 1.2. 
Proof of Theorem 1.2 Since $\{S, N\}$ generates a solvable Lie algebra $\mathfrak{s}$, and $\mathbb{F}$ is algebraically closed, it follows from Lie's Theorem that we can assume $S, N \in \mathfrak{s} \subset \mathfrak{t}$, and since $N$ is nilpotent, $N \in \mathfrak{t}^{\prime}$.

We will prove (i) by induction. Assume (i) is true for $S_{n}$ and $N_{n}$ and let us suppose that $\left[S_{n}, N_{n}\right] \neq 0$. Since $\lambda_{n} \neq 0$, we have $\left(N_{n}\right)_{\lambda_{n}} \in \mathfrak{t}^{\prime}$, and hence $N_{n+1}$ is nilpotent. It follows from Lemma 2.1 that $S_{n+1}$ is semisimple and $S_{n+1}, N_{n+1} \in \mathfrak{s}$. This proves (i).

It follows from Lemma 2.4 that

$$
\gamma_{S_{n+1}}\left(N_{n+1}\right)=\gamma_{S_{n}+\left(N_{n}\right)_{\lambda_{n}}}\left(N_{n}-\left(N_{n}\right)_{\lambda_{n}}\right)<\gamma_{S_{n}}\left(N_{n}\right)
$$

in the lexicographical order. This implies that there exists $n_{0}$ such that $\gamma_{S_{n_{0}}}\left(N_{n_{0}}\right)=0$ and hence $\left[S_{n_{0}}, N_{n_{0}}\right]=0$. This proves (ii), and it is clear $A=S_{n_{0}}+N_{n_{0}}$ is the JordanChevalley decomposition of $A$.

Finally, let $\pi: \mathfrak{s} \rightarrow \mathfrak{g l}(V)$ be a representation such that $\pi(S)=\pi\left(S_{0}\right)$ is semisimple and $\pi(N)=\pi\left(N_{0}\right)$ is nilpotent. Since $\pi$ is a representation, if $N_{n}=\sum_{\lambda \in \Lambda_{n}}\left(N_{n}\right)_{\lambda}$ is the $\operatorname{ad}_{\mathfrak{s}}\left(S_{n}\right)$-decomposition of $N_{n}$, then

$$
\pi\left(N_{n}\right)=\sum_{\lambda \in \Lambda_{n}} \pi\left(\left(N_{n}\right)_{\lambda}\right)
$$

is the $\operatorname{ad}_{\pi(\mathfrak{s})}\left(\pi\left(S_{n}\right)\right)$-decomposition of $\pi\left(N_{n}\right)$. Therefore, assuming that $\pi\left(S_{n}\right)$ is semisimple and $\pi\left(N_{n}\right)$ is nilpotent, it follows, just as above, that $\pi\left(S_{n+1}\right)$ is semisimple and $\pi\left(N_{n+1}\right)$ is nilpotent. This implies that $\pi(A)=\pi\left(S_{n_{0}}\right)+\pi\left(N_{n_{0}}\right)$ is the JordanChevalley decomposition of $\pi(A)$.

Proof of Theorem 1.1 Theorem 1.2 shows that Theorem 1.1 is true when $\mathbb{F}$ is algebraically closed, since in this case, Lie's Theorem allows us to assume that $\mathfrak{s} \subset \mathfrak{t}$.

In general, let $\overline{\mathbb{F}}$ be an algebraic closure of $\mathbb{F}$. Suppose $A, S, N \in \mathfrak{s}$, where $A=$ $S+N, S$ is semisimple, and $N$ is nilpotent. Let $A=S^{\prime}+N^{\prime}$ be the JCD of $A$ in $\mathfrak{g l}(n, \mathbb{F})$, as ensured in $[\mathrm{HK}, \$ 7.5]$. The minimal polynomial of $S^{\prime}$, say $p$, is a product of distinct monic irreducible polynomials over $\mathbb{F}[\mathrm{HK}, \S 7.5]$. Since $\mathbb{F}$ has characteristic 0 , it follows that $p$ has distinct roots in $\overline{\mathbb{F}}$, whence $S^{\prime}$ is diagonalizable over $\overline{\mathbb{F}}$. Therefore, $A=S^{\prime}+N^{\prime}$ is the JCD of $A$ in $\mathfrak{g l}(n, \overline{\mathbb{F}})$. Let $\overline{\mathfrak{s}}$ be the $\overline{\mathbb{F}}$-linear span of $\mathfrak{s}$ in $\mathfrak{g l}(n, \overline{\mathbb{F}})$. Then $\overline{\mathfrak{s}}$ is a solvable subalgebra of $\mathfrak{g l}(n, \overline{\mathbb{F}})$. As the theorem is true over $\overline{\mathbb{F}}$, we infer $S^{\prime}, N^{\prime} \in \overline{\mathfrak{s}}$. Thus, $S^{\prime}, N^{\prime} \in \mathfrak{g l}(n, \mathbb{F}) \cap \overline{\mathfrak{s}}=\mathfrak{s}$. This completes the proof of Theorem 1.1.

\section{Jordan-Chevalley Decomposition in a Lie Algebra}

Theorem 3.1 An element $x$ of a Lie algebra $\mathfrak{g}$ has an abstract JCD if and only if $x$ belongs to the derived algebra $[\mathfrak{g}, \mathfrak{g}]$, in which case the semisimple and nilpotent parts of $x$ also belong to $[\mathfrak{g}, \mathfrak{g}]$.

Necessity This is clear, since any linear map from $\mathfrak{g}$ to $\mathfrak{g l}(V) \operatorname{such}$ that $\operatorname{dim} \pi(\mathfrak{g})=1$, and $\pi([\mathfrak{g}, \mathfrak{g}])=0$ is a representation.

Sufficiency By Ado's theorem, we can assume that $\mathfrak{g}$ is a Lie algebra of matrices. Fix a Levi decomposition $\mathfrak{g}=\mathfrak{g}_{s} \ltimes \mathfrak{r}$ and let $\mathfrak{n}=[\mathfrak{g}, \mathfrak{r}]$. We know that $\mathfrak{n}$ is nilpotent (see [FH, Lemma C.20]). If $x \in[\mathfrak{g}, \mathfrak{g}]$, then $x=a+r$ for unique $a \in \mathfrak{g}_{s}$ and $r \in \mathfrak{n}$. If $a=a_{s}+a_{n}$ is the JCD of the matrix $a$, since $\mathfrak{g}_{s}$ is semisimple, it follows that $a_{s}, a_{n} \in \mathfrak{g}_{s}=\left[\mathfrak{g}_{s}, \mathfrak{g}_{s}\right]$ (see, for instance, $[\mathrm{Hu}, \$ 6.4]$ ). Let $\mathfrak{s}=\mathbb{F} a_{s} \oplus \mathbb{F} a_{n} \oplus \mathfrak{n} \subset[\mathfrak{g}, \mathfrak{g}]$. Since $[\mathfrak{s}, \mathfrak{s}] \subset \mathfrak{n}$ and $\mathfrak{n}$ 
is nilpotent, we obtain that $\mathfrak{s}$ is a solvable Lie algebra. We now apply Theorem 1.1 to the Lie algebra $\mathfrak{s}$ with $S=a_{s}, N=a_{n}+r$. We obtain that if $x=S^{\prime}+N^{\prime}$ is the JCD of $x$, then $S^{\prime}, N^{\prime} \in \mathfrak{s} \subset[\mathfrak{g}, \mathfrak{g}]$.

Finally, let $\pi: \mathfrak{g} \rightarrow \mathfrak{g l}(V)$ be a representation of $\mathfrak{g}$. Since $r \in \mathfrak{n}$, it follows that $\pi(r)$ is nilpotent (see [FH, Lemma C.19] or [B1, Ch.1, \$5]). Since $\mathfrak{g}_{s}$ is semisimple, $\pi(S)=\pi\left(a_{s}\right)$ is semisimple and $\pi\left(a_{n}\right)$ is nilpotent. Since $\mathfrak{s}$ is solvable, it follows from Lie's Theorem that $\pi(N)=\pi\left(a_{n}+r\right)$ is nilpotent. It follows from Theorem 1.2 (applied over a field extension of $\mathbb{F}$ ) that $\pi(x)=\pi\left(S^{\prime}\right)+\pi\left(N^{\prime}\right)$ is the JCD of $\pi(x)$.

\section{References}

[B1] N. Bourbaki, Lie Groups and Lie Algebras: chapters 1-3. Springer-Verlag, Berlin, 1989.

[B2] L Lie Groups and Lie Algebras: chapters 7-9. Springer-Verlag, Berlin, 2005.

[CS] L. Cagliero and F. Szechtman, Jordan-Chevalley decomposition in finite dimensional Lie algebras. Proc. Amer. Math. Soc. 139(2011), no. 11, 3909-3913. http://dx.doi.org/10.1090/S0002-9939-2011-10827-X

[CS2] The classification of uniserial $\mathfrak{s l}(2) \rtimes V(m)$-modules and a new interpretation of the Racah-Wigner 6j-symbol. J. Algebra 386(2013), 142-175. http://dx.doi.org/10.1016/j.jalgebra.2013.03.022

[CS3] On the theorem of the primitive element with applications to the representation theory of associative and Lie Algebras. Canad. Math. Bull. 57(2014), no. 4, 735-748. http://dx.doi.org/10.4153/CMB-2013-046-9

[FH] W. Fulton and J. Harris, Representation theory: A first course. Graduate Texts in Mathematics, 129, Readings in Mathematics, Springer-Verlag, New York, 1991. http://dx.doi.org/10.1007/978-1-4612-0979-9

[HK] K. Hoffman and R. Kunze, Linear algebra. Second ed., Prentice-Hall, New Jersey, 1971.

[Hu] J. E. Humphreys, Introduction to Lie algebras and representation theory. Graduate Texts in Mathematics, 9, Springer-Verlag, New York-Berlin, 1978.

[Ki] K.-T. Kim, Criteria for the existence of a Jordan-Chevalley-Seligman decomposition. J. Algebra 424(2015), 376-389. http://dx.doi.org/10.1016/j.jalgebra.2014.09.028

CIEM-CONICET, FAMAF-Universidad Nacional de Córdoba, Córdoba, Argentina

e-mail: cagliero@famaf.unc.edu.ar

Department of Mathematics and Statistics, Univeristy of Regina, Regina, SK

e-mail: fernando.szechtman@gmail.com 